\title{
Modern Strategy and Biological Fate of Acute Pneumonia
}

\author{
Igor Klepikov, $M D^{*}$
}

Pediatric Surgeon, WA, USA

The dynamics of any disease development is determined by the presence of biological rules and laws that exist in nature. Due to the influence of such stereotypes, the originality of clinical manifestations of a particular pathology is formed. This is an axiom of biological rhythms of living nature, which must be taken into account when conducting scientific research and searching for interpretations of the clinical picture of various diseases. As a result, a differentiated view of the uniqueness of the mechanism of development and clinical manifestations of each individual disease is formed, which is the basis of the principles of diagnosis and treatment.

The basis of the evolution of medical knowledge and concepts is well known and is unlikely to cause anyone serious objections, as are the obvious differences between pneumococcal meningitis and pneumococcal pneumonia, or between staphylococcal sinusitis and staphylococcal soft tissue abscess, is not it? However, the evidence of general principles in the study of various pathologies does not mean that these approaches are always strictly followed. Therefore, when the results are analyzed and interpreted based on common signs or similarities of complications of various diseases, the quality of such analysis, in my opinion, decreases, to the point of distortion of the results. A separate analytical approach is especially important when considering processes with impaired hemodynamics, depending on the location of the main focus within the small or large circle of blood circulation.

In connection with the latter features, it is necessary to pay special attention to acute pneumonia (AP). This disease consistently occupies one of the leading places in the summary medical statistics for such important indicators as morbidity and mortality. Characterized by a severe course, AP has a high incidence of complications, among which septic shock (SS) is considered the most critical. However, when analyzing the observations of SS in patients with $\mathrm{AP}$, its manifestations and mechanisms of development are completely identified with a similar complication in other fundamentally different diseases.

The weak point of such analytical work on the problem of SS is precisely the combination of seemingly similar complications of various diseases [1-3]. These links to publications on this topic are taken only as examples, since there are many more such publications, and they all have a common research methodology. First of all, we are talking about the fact that the unique features of AP development, unlike other inflam- matory processes, are not taken into account when evaluating the results of treatment of this terminal condition. Usually, the text of publications does not indicate the composition of patients and the distribution of this severe complication by nosology, since the authors consider SS to be an identical complication regardless of the root cause. But the materials of intensive care units always include a large number of observations of complicated forms of $\mathrm{AP}$, and indirect signs of such analysis indicate a high proportion of such patients with SS. In this regard, a separate analysis of this group of patients and fundamental scientific data on the anatomical and functional features of the lungs can significantly affect the understanding of the reasons for the low effectiveness of SS treatment in AP.

The lungs are the only organ that provides respiratory function in the body, so it is logical that gas exchange disorders are always taken into account in their diseases. At the same time, the so-called non-respiratory functions of the lungs are almost always overlooked. Among these natural vocations of the lung tissue, one of the most important functions is to participate in blood circulation and especially in its regulation.

It is well known that the volume of blood that the left ventricle throws into the systemic circulation should be exactly equal to the volume that enters the lungs. This strict rule of normal blood circulation and vital activity of the body is supported by a significantly lower pressure in the vessels of the small circle of blood circulation. Violation of this balance leads to extreme functional disorders in the cardiovascular system, and in order to eliminate the resulting imbalance, we do not need to look for suitable ways to correct it. In our body, there are various natural mechanisms that are aimed at automatically correcting dangerous situations.

One of these mechanisms was discovered and described in the thirties of the last century by Schwiegk [4]. This is a reflex on the part of the baroreceptors of the pulmonary ves-

*Corresponding author: Igor Klepikov, Professor Retired, Pediatric Surgeon, 2116 27th St. NE Renton, WA, USA

Accepted: July 06, 2020

Published online: July 08, 2020

Citation: Klepikov I (2020) Modern Strategy and Biological Fate of Acute Pneumonia. Res Rev Infect Dis 3(2):78-80 
sels, which is triggered in the event of an increase in blood pressure in them. Physiologists often call this reflex unloading. Thus, in the case of increased pressure in the pulmonary vessels, there is a reflex decrease in systemic blood pressure, slowing of the heart rate, increased blood filling of the spleen and vasodilation in the skeletal muscles. The expansion of peripheral vessels reduces blood flow to the small circle of blood circulation and, consequently, unloads the pulmonary capillaries and protects the lungs from edema.

To date, the presence of such a protective-adaptive mechanism has been sufficiently studied and known, and its action can be further enhanced by vasoactive factors that are released and activated in pathological situations. The occurrence of such a reaction in blood flow disorders in the small circle of circulation and its role in the dynamics of pulmonary pathology are not always taken into account when interpreting the features of the course of diseases. The most common causes of such hemodynamic rearrangement are discussed in patients with pulmonary embolism [5-7], the clinical manifestations of which can vary from an asymptomatic picture to sudden death, but it is the latter sudden outcome that arouses interest in its causes. At the same time, it should be noted that there is no complete relationship and correlation between the size of the embolus and its clinical consequences, which are more a manifestation of individual characteristics.

$\mathrm{AP}$, regardless of its etiology, is an inflammatory process with significant damage to vascular structures $[8,9]$, which cannot leave the baroreceptors of the lung vessels indifferent. Therefore, the mechanism of regulation of systemic blood flow described above will be unavoidable in patients with $A P$, but the speed and manifestation of this transformation will not be as instantaneous as in embolism, and the question is how quickly inflammation develops in the lungs and what are the adaptive and compensatory capabilities of a particular organism.

Aggressive onset of AP leaves the patient less time and chances for adaptation, and the resulting picture of changes in peripheral blood flow in the most severe cases corresponds to the picture of shock. The dominant microbial concept of the disease for many years interprets such a clinic in AP patients as SS [1-3,10], despite the fact that this diagnosis in most cases has no bacteriological confirmation $[1,11]$.

However, it is not only the lack of justification and confirmation of such a diagnosis, but also the fact that this diagnosis determines the direction of efforts to provide medical care to such patients. And since the main goal of treatment remained the pathogen of the disease, the principles of assistance for SS of various Genesis were automatically transferred to patients with AP.

Interpretation of the nature of shock in AP is crucial for choosing ways to eliminate it. The authors consider the nature of shock as septic, so they recommend, first of all, to direct therapeutic efforts to suppress the pathogen and replenish the volume of circulating blood by intravenous infusions. Empirical use of broad-spectrum antibiotic therapy is recommended due to the lack of information about the pathogen in the vast majority of patients with SS. The assumption that treatment results will improve if antibiotics are applied as early as possible also has no noticeable effect on them. In other words, antibacterial therapy, which is considered the main medical care, does not give the significant effect that is expected from it.

At the same time, intravenous infusions for SS are designed to eliminate hypovolemia, increase cardiac output, and thereby improve tissue perfusion. However, if there is a pathological process in the lung tissue, such therapy will increase, first of all, the blood flow to the pulmonary vessels and increase the pressure in them. The direct consequence of such treatment will, on the contrary, further decrease in systemic blood pressure, because the reflex of unloading from the baroreceptors of the pulmonary vessels does not disappear anywhere and continues to act, does it not? Therefore, the experience of intravenous infusions in such situations did not allow the authors to justify specific recommendations for this type of treatment, but rather to emphasize the likelihood of its negative impact on hemodynamics in such patients [3]. Negative aspects of intravenous fluids are reflected in the discussion of this section of therapy.

First, it was noted that bolus therapy increases mortality in the first hour of resuscitation. Secondly, the attention of readers is drawn to the need for careful prescribing of liquid therapy and its immediate termination when signs of overload appear. Third, it is recommended to give preference to the infusion of crystalloids over colloidal solutions, although from the point of view of the goal, the use of colloids is preferable to replenish the volume of circulating blood in order to improve tissue perfusion. Finally, the authors note a high percentage of so-called refractory types of shock that do not respond properly to the introduction of fluids and require additional vasopressors [3].

Thus, the analysis of extensive clinical experience and generalization of its results show that the most priority types of medical care for patients with SS (antibiotic therapy and liquid therapy) do not have the expected effect to achieve which they are used. In this situation, the need for a thorough revision of the basic ideology of these States and justification of the goals and objectives of new therapeutic approaches becomes quite obvious. First of all, this applies to the allocation of patients with lung inflammation in a separate analytical group. This need is due to the peculiarities of the pathogenesis of AP and, in particular, the reaction of the patient's body in response to such a treatment procedure as intravenous fluid injection.

For many years, the existing discrepancies between theoretical assumptions, on the one hand, and the actual data and results of AP treatment, on the other, indicate the need to revise the concept of this disease. Well-established ideas about nature have been formed over the years under the influence of a hypnotic belief in antibacterial drugs for therapy. At the same time, the main paradox of this problem is that the more the effectiveness of antibacterial therapy decreased and the number of resistant strains increased, the more persistent became the purposeful and empirical attempts to suppress bacterial pathogens, and the disease itself in recent years has 
become unfairly regarded as infectious. Despite the efforts made in the field of antibacterial therapy, the effectiveness of treatment continues to decline. Against this background, the current state of the problem is significantly complicated by an increase in the frequency of viral lung inflammation, in which the usual therapeutic approach with the leading role of antibiotics generally becomes impractical.

Continuing to consider shock in AP as a result of generalization of infection from the focus of inflammation, modern concepts give various assumptions in an attempt to explain such a contradictory and undoubted fact as negative results in most patients of bacteriological blood tests. Such studies in patients with severe forms of AP are positive only in 10$12 \%$ of cases, which include not only SS, but also sepsis and bacteremia [12-14]. The most common explanation for such discrepancies between the septic nature of shock and the absence of its main diagnostic confirmation is the prior use of antibiotics [15]. However, in this case, a logical question arises: Why, despite such effective antibacterial therapy, does SS develop?

Another assumption about the reasons for the discrepancy between the assessment of the nature of shock in AP and its bacteriological confirmation is a rather strange hypothesis about its viral etiology. Emphasizing the undoubted bacterial nature of AP when analyzing treatment results, the authors consider the viral nature of shock as a possible cause of negative bacteriological tests in such patients [15]. This misconception has, from my point of view, a very obvious origin, as a result of the long-term didactic influence of a narrow antibacterial concept of the disease. It is easy to see that all explanations of facts that do not correspond to theoretical assumptions are aimed only at searching for possible pathogens. The probability of other reasons for the existence of contradictions is not even discussed. The paradox of such explanations is that such a high frequency of negative bacteriological blood tests in SS should, first of all, raise doubts about its septic origin. This contradiction should be studied from other points of view, if only because the presence of shock is really confirmed by severe hemodynamic disorders in these patients against the background of sterile blood cultures $[2,3]$.

The mechanism underlying the unloading of the small circle of blood circulation in the case of rapid and abrupt changes in its tissue structures explains the uniqueness of shock in patients with AP. This form of circulatory disorder was previously described by the author as a pulmonal shock, and its elimination requires completely different therapeutic approaches in contrast to other shock states. The spectrum of such circulatory disorders has an infinite number of individual options, starting with an asymptomatic clinic. The described fragment of AP pathogenesis was used as one of the justifications for pathogenetic approaches to treatment. The accuracy of this choice was confirmed by both objective tests and the results of the patient's treatment [16], and the data obtained, from my point of view, are more interesting today than at the time of completion of the work.

In this context, there is only one reason that requires a thorough and comprehensive re-evaluation of the AP strategy. Detailed tactical decisions will be the next step in this direction. At present, when practical medicine around the world is faced with a huge flow of severe patients with viral pneumonia, and the effectiveness of medical care does not give confidence and optimism, the current situation requires, first of all, a critical and reasoned revision of existing ideas about the pathogenesis of this disease. Such biological patterns and stereotypes as processes of acute inflammation and non-respiratory lung functions are waiting for their rightful place in the interpretation of the development and manifestations of AP. Their role and impact on the disease clinic and treatment outcomes will continue to operate regardless of prevailing perceptions and preferences.

\section{References}

1. Schlapbach LJ, Straney L, Alexander J, et al. (2015) Mortality related to invasive infections, sepsis, and septic shock in critically ill children in Australia and New Zealand, 2002-13: A multicentre retrospective cohort study. Lancet Infect Dis 15: 46-54.

2. Singer M, Deutschman CS, Seymour CW, et al. (2016) The third international consensus definitions for sepsis and septic shock (Sepsis-3). JAMA 315: 801-810.

3. Weiss SL, Peters MJ, Alhazzani W, at al. (2020) Surviving sepsis campaign international guidelines for the management of septic shock and sepsis-associated organ dysfunction in children. Pediatr Crit Care Med 21: e52-e106.

4. Schwiegk H (1935) Der Lung enentlastungsreflex. Pflügers Arch Ges Physiol 236: 206-219.

5. Elliott CG (1992) Pulmonary physiology during pulmonary embolism. Chest 101: 163S-171S.

6. Lualdi JC, Goldhaber SZ (1995) Right ventricular dysfunction after acute pulmonary embolism: Pathophysiologic factors, detection, and therapeutic implications. Am Heart J 130: 1276-1282.

7. Turetz M, Sideris AT, Friedman OA, et al. (2018) Epidemiology, pathophysiology, and natural history of pulmonary embolism. Semin Intervent Radiol 35: 92-98.

8. Cotran RS, Kumar V, Fausto N, et al. (2005) Robbins and cotran pathologic basis of disease. Elsevier Saunders, St. Louis, Missouri, 749.

9. Pahal P, Sharma S (2020) Typical Bacterial Pneumonia. In: StatPearls [Internet]. StatPearls Publishing, Treasure Island (FL).

10. Schlapbach L, Weiss SL, Wolf J (2019) Reducing collateral damage from mandates for time to antibiotics in pediatric sepsis-primum non nocere. JAMA Pediatr 173: 409-410.

11. Weiss SL, Fitzgerald JC, Pappachan J, et al. (2015) Global epidemiology of pediatric severe sepsis: The sepsis prevalence, outcomes, and therapies study. Am J Respir Crit Care Med 191: 1147-1157.

12. AJ Morgan, AJ Glossop (2016) Severe community-acquired pneumonia. BJA Education 16: 167-172.

13. Sanjay Sethi (2019) Community-acquired pneumonia. Merck Manual, Professional version.

14. GW Waterer, MW Quasney, RM Cantor, et al. (2001) Septic shock and respiratory failure in community-acquired pneumonia have different TNF polymorphism associations. Am J Respir Crit Care Med 163: 1599-1604.

15. Lin GL, McGinley JP, Drysdale SB, et al. (2018) Epidemiology and immune pathogenesis of viral sepsis. Front Immunol 9: 2147.

16. Igor Klepikov (2019) The role and importance of biological stereotypes in the pathogenesis of acute pneumonia. EC Pulmonology and Respiratory Medicine 83: 239-246. 